\title{
An improved algorithm for fluid-structure interaction of high-speed trains under crosswind
}

\author{
Tian LI*, Jiye ZHANG, Weihua ZHANG \\ Traction Power State Key Laboratory, Southwest Jiaotong University, Chengdu 610031, China
}

\begin{abstract}
Based on the train-track coupling dynamics and high-speed train aerodynamics, this paper deals with an improved algorithm for fluid-structure interaction of high-speed trains. In the algorithm, the data communication between fluid solver and structure solver is avoided by inserting the program of train-track coupling dynamics into fluid dynamics program, and the relaxation factor concerning the load boundary of the fluid-structure interface is introduced to improve the fluctuation and convergence of aerodynamic forces. With this method, the fluid-structure dynamics of a highspeed train are simulated under the condition that the velocity of crosswind is $13.8 \mathrm{~m} / \mathrm{s}$ and the train speed is $350 \mathrm{~km} / \mathrm{h}$. When the relaxation factor equals 0.5 , the fluctuation of aerodynamic forces is lower and its convergence is faster than in other cases. The side force and lateral displacement of the head train are compared between off-line simulation and co-simulation. Simulation results show that the fluid-structure interaction has a significant influence on the aerodynamics and attitude of the head train under crosswind conditions. In addition, the security indexes of the head train worsen after the fluid-structure interaction calculation. Therefore, the fluid-structure interaction calculation is necessary for high-speed trains.
\end{abstract}

Key words: high-speed train; fluid-structure interaction; crosswind; aerodynamics; relaxation factor

(C) 2011 JMT. All rights reserved.

\section{Introduction}

$\mathrm{H}$ igh-speed transportation is the new direction of modern railway transportation [1]. High-speed train aerodynamics and train-track coupling dynamics, the indispensable parts of high-speed transportation system, are mutually coupled and influenced. The action of aerodynamic forces will change the running attitude of the train, and consequently the running attitude will affect the flow field around train. Thus, the aerodynamic forces of the train will change, and the train system will be in a particular state of coupling vibration under this mutual feedback.

Strong crosswind seriously affects the running security of high-speed trains. The train derailments and overturns happen because of strong crosswind [2-5]. Many researchers [6-11] have analyzed the running security of trains under crosswinds. There are mainly two calculation methods of high-speed dynamics under crosswind conditions: off-line simulation method, and cosimulation method. The former is as follows: the aerodynamic forces of the train under crosswind conditions are calculated first, and then the dynamic responses of

Received May 30, 2011; revision accepted Jun.18, 2011

*Corresponding author. E-mail: litian3408@163.com (T. LI) doi: 10.3969/j.issn.2095-087X.2011.02.001 the train are calculated with the train dynamic model, on which the static aerodynamic forces are loaded. This method neglects the change of attitude caused by the action of aerodynamic forces, and does not reflect the essence of the aerodynamics and train dynamics. In the co-simulation, the high-speed train aerodynamics and train-track dynamics are calculated alternatively during the interaction; namely, the interaction effect between the high-speed train aerodynamics and train-track dynamics is considered. Yang et al. [10] and Cui et al. [11] performed a co-simulation between high-speed train aerodynamics and train dynamics through parameter transfer and synchronization control, but they neglected the influence of track structures on the system [11]. In Refs. [10-11], the train dynamic solver is in a state of either calculating or waiting, which consumes too much memory and resources. In addition, the information exchange on the interface from one time step to the next time step in an alternating fashion can easily cause energy dissipation.

In this paper, a co-simulation algorithm was improved by (1) inserting the program of train-track dynamics into the program of computational fluid dynamics to avoid the data communication between fluid and structure solvers, and (2) introducing the relaxation factor to improve the information exchange on the interface 
between aerodynamics and train dynamics. With the improved algorithm, we conducted a co-simulation between aerodynamics and train-track dynamics for a train running at a speed of $350 \mathrm{~km} / \mathrm{h}$ under a crosswind of velocity $13.8 \mathrm{~m} / \mathrm{s}$, and analyzed the fluid-structure dynamics of the high-speed train.

\section{Governing equations}

\subsection{Governing equations of fluid dynamics}

When the high-speed train is running under crosswind, its flow field can be considered as a three-dimensional transient viscous turbulent flow. When the running speed is lower than $400 \mathrm{~km} / \mathrm{h}$, the flow field around the train can be considered as an incompressible flow. The standard $k$ $\varepsilon$ two-equation model is adopted and the equations of incompressible flow [9] are written as

$$
\frac{\partial(\rho \boldsymbol{\phi})}{\partial t}+\operatorname{div}(\rho \boldsymbol{u} \boldsymbol{\phi})=\operatorname{div}(\Gamma \operatorname{grad} \boldsymbol{\phi})+\boldsymbol{S},
$$

where $t$ is time, $\rho$ is the air density, $\boldsymbol{u}$ is the velocity vector, $\phi$ is flow flux, $S$ is the source term, and $\Gamma$ is the diffuse coefficient.

\subsection{Equations of train-track coupling dynamics}

The train-track coupling dynamics mainly include vehicle dynamics, track dynamics, and wheel-rail contact. It is assumed that the body, bogies, and wheelsets are rigid and that their elastic deformations can be neglected. The track system is considered as a continuously distributed spring-damper model with a two-mass (sleeper and ballast) and three-layer (rail-sleeperballast-bed) structure. The equations of the train-track coupling dynamics [9] are written as

$$
\boldsymbol{M} \ddot{\boldsymbol{X}}+\boldsymbol{C} \dot{\boldsymbol{X}}+\boldsymbol{K} \boldsymbol{X}=\boldsymbol{F},
$$

where $\boldsymbol{M}, \boldsymbol{C}$, and $\boldsymbol{K}$ are the mass, damp, and stiffness matrixes of train-track system, respectively; $\boldsymbol{X}, \dot{\boldsymbol{X}}$, and $\ddot{X}$ are the generalized displacement, velocity, and acceleration vectors of the system, respectively; and $\boldsymbol{F}$ is the generalized load vector including the rail excitation and aerodynamic forces load.

\section{Improved algorithm for fluid-structure interaction}

\subsection{Technique for solving train-track dynamics equation}

Based on the theory of train-track coupling dynamics, the program of train-track coupling dynamics is written with FORTRAN and verified to be reliable [9].
The train-track dynamics equations are solved with the Zhai's method [12]. Introducing two integral parameters $\varphi$ and $\psi$, we construct the new explicit integral format as:

$$
\left\{\begin{array}{l}
\boldsymbol{X}_{n+1}=\boldsymbol{X}_{n}+\dot{\boldsymbol{X}}_{n} \Delta t+\left(\frac{1}{2}+\psi\right) \ddot{\boldsymbol{X}}_{n} \Delta t^{2}-\psi \ddot{\boldsymbol{X}}_{n-1} \Delta t^{2}, \\
\dot{\boldsymbol{X}}_{n+1}=\dot{\boldsymbol{X}}_{n}+(1+\varphi) \ddot{\boldsymbol{X}}_{n} \Delta t-\varphi \ddot{\boldsymbol{X}}_{n-1} \Delta t
\end{array}\right.
$$

where $\Delta t$ is the integral interval, and subscript $n$ means the time iteration step.

The form of (3) at time $t=(n+1) \Delta t$ is

$$
\boldsymbol{M} \ddot{\boldsymbol{X}}_{n+1}+\boldsymbol{C} \dot{\boldsymbol{X}}_{n+1}+\boldsymbol{K} \boldsymbol{X}_{n+1}=\boldsymbol{F}_{n+1},
$$

Substituting (4) into (3), we can calculate $\ddot{\boldsymbol{X}}_{n+1}$.

\subsection{Mesh renewing technique}

The spring analogy method [12] and the re-mesh method are adopted to renew the mesh. When the spring analogy method fails, the re-mesh method is adopted. Spring analogy method is a simple but efficient method among mesh deforming methods. In this method, each edge of the grid is modeled as a linear tension spring. The spring stiffness for a given edge $i-j$ is taken to be inversely proportional to the length of the edge as

$$
K_{i j}=\frac{1}{r_{i j}}=\frac{1}{\left\|\boldsymbol{r}_{i}-\boldsymbol{r}_{j}\right\|},
$$

where $r_{i j}$ is the distance between node $i$ and $j$, and $\boldsymbol{r}_{i}$ is the position vector of node $i$.

The displacements of grid points is solved by

$$
\sum_{j}^{N_{i}} K_{i j} \Delta \boldsymbol{r}_{j}=0,
$$

where $N_{i}$ is the total number of the nodes connected with node $i$, and $\Delta r_{j}$ is the displacement of node $j$. The summations are performed over all the edges of quadrilaterals with node $i$ as an end point, $i=1,2, \cdots, n$.

The new locations of the nodes are determined by

$$
\overline{\boldsymbol{r}_{i}}=\boldsymbol{r}_{i}+\Delta \boldsymbol{r}_{i}
$$

\subsection{Solution strategies}

Fig. 1 shows the co-simulation method of high-speed train fluid-structure interaction. The process of the method is described as follows: first, calculate the aerodynamic forces of train under crosswind until the forces reach a relatively steady state, and then the iteration between aerodynamics and train-track coupling dynamics begins. At each iteration, the transient aerodynamic forces are transferred from the fluid solver to the train dynamic 
model, and the responses of train dynamics are calculated with this model. Then the attitudes are transferred to the fluid solver and the aerodynamic forces are calculated under the train attitude. In this method, the state of train dynamic solver is either calculating or waiting, causing waste of memory and resources. In addition, the load boundary of aerodynamics and dynamics is very simple, which may cause energy dissipation.

Fig. 2 shows an improved co-simulation method of high-speed train fluid-structure interaction. The fluidstructure solver includes the aerodynamic solver and train-track coupling dynamic solver, and the latter is inserted into the aerodynamic solver through interface. Thus, the data communication between fluid and structure solvers is avoided, and the train-track coupling dynamic solver does not have to wait when the fluid solver is calculating. The relaxation factor is introduced for renewing the boundary of aerodynamics and dynamics. The aerodynamic forces loaded to the train-track dynamics model at time $i+1$ is no more than the forces at time $i$, which is predicted by the aerodynamic forces and their velocities at time $i$.

It is assumed that the aerodynamic forces $f_{i-1}$ and $f_{i}$ are calculated by the fluid solver at time $i-1$ and $i$, respectively, and the time interval is $\Delta t$. As the $\Delta t$ is very small, the velocity of aerodynamic forces at time $i$ is

$$
\dot{f}_{i}=\frac{f_{i}-f_{i-1}}{\Delta t} .
$$

Thus, the predicted aerodynamic force at time $i+1$ is

$$
f_{i+1}=f_{i}+\Delta t \times \dot{f}_{i}=2 f_{i}-f_{i-1} .
$$

By introducing the relaxation factor $\lambda$, the predicted aerodynamic force $\tilde{f}_{i+1}$, which is loaded to the traintrack dynamic model at time $i+1$, is expressed as follows:

$$
\tilde{f}_{i+1}=(1-\lambda) f_{i}+\lambda f_{i+1}=(1+\lambda) f_{i}-\lambda f_{i-1} .
$$

When $\lambda=0$, the aerodynamic force loaded to the traintrack dynamic model at time $i+1$ equals $f_{i}$. Under this condition, the method is similar to the method in [10-11].

\section{Simulation}

\subsection{Computational domain and boundary}

In this section, we describe the fluid-structure problem of the high-speed train under crosswind. The schematic of the computational domain is shown in Fig. 3. The computational range is $350 \mathrm{~m}$ in length, $90 \mathrm{~m}$ in width and $60 \mathrm{~m}$ in height. The distance from the inlet boundary to the nose of the head train is $100 \mathrm{~m}$, and the distance from the outlet boundary to the nose of the tail train is $175 \mathrm{~m}$. Velocity inlet condition and traction-free condition are preset at the inlet boundary and outflow boundary, respectively. In addition, a no-slip condition

\section{Aerodynamics}

Train-track coupling dynamics
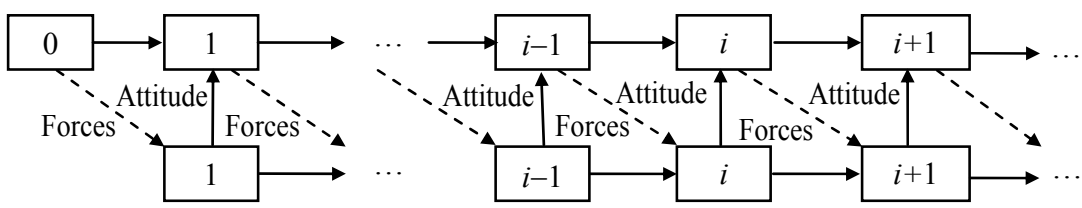

Fig. 1 Co-simulation method of high-speed train fluid-structure interaction
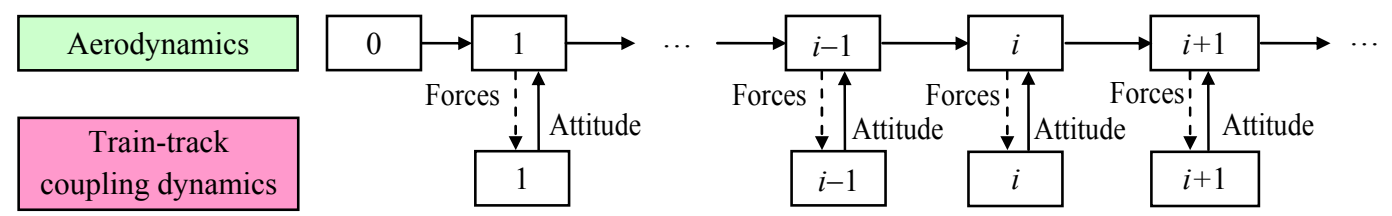

Fig. 2 Improved co-simulation method of high-speed train fluid-structure interaction

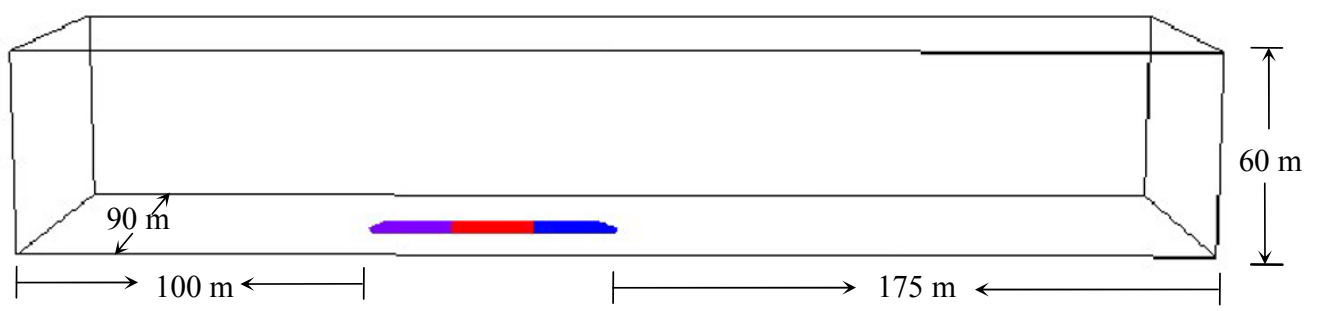

Fig. 3 Schematic of computational domain 
and symmetry condition are specified as the train surface and top boundaries, respectively. The slip condition is adapted to the wall boundary.

The train consists of three cars, including head train, middle train and tail train, with the bulge (such as the pantograph) ignored. The iteration time steps of the fluid dynamic and the train-track dynamic are $2.0 \times 10^{-3} \mathrm{~s}$ and $5.0 \times 10^{-5} \mathrm{~s}$, respectively. In this paper, calculations are carried out for a high-speed train with a speed of $350 \mathrm{~km} / \mathrm{h}$ and the crosswind velocity of $13.8 \mathrm{~m} / \mathrm{s}$; that is, the combination velocity equals $98.2 \mathrm{~m} / \mathrm{s}$ and the yaw angle is $8.08^{\circ}$.

\subsection{Influence of relaxation factor}

The load boundary of fluid-structure interface, with different values of the relaxation factor, may affect the energy dissipation. In this section, three different relaxation factors $(\lambda=0.0,0.5$ and 1.0$)$ are chosen to analyze the effect.

Fig. 4 shows the side force and lift force curves calculated with different relaxation factors. The change curves of forces are almost the same, except some differences in the amplitudes of side force and lift force

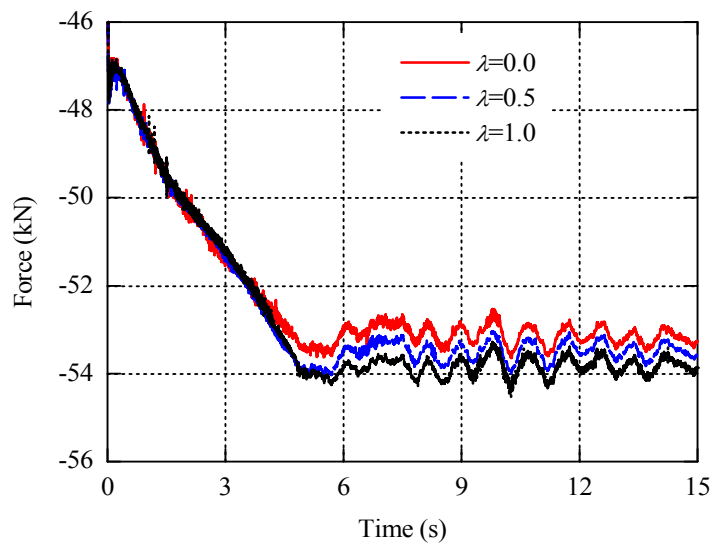

(a) Side force

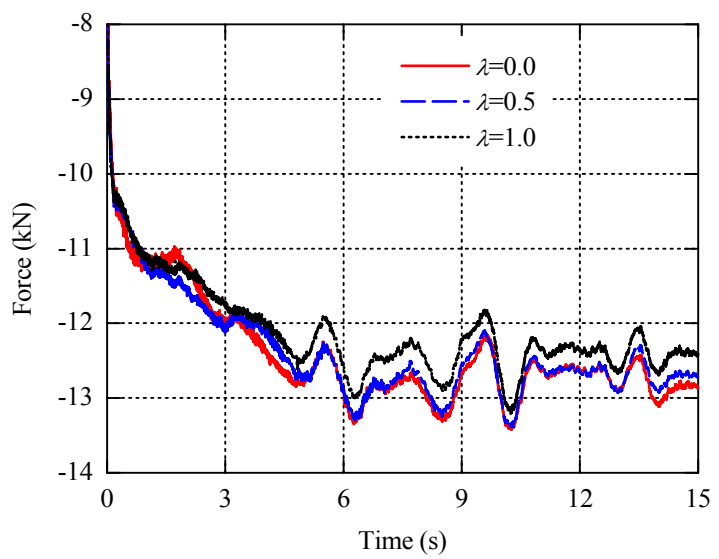

(b) Lift force

Fig. 4 Side force and lift force with different $\lambda$ with different relaxation factors. The amplitude of side force becomes larger with an increase in relaxation factor. However, the opposite is true with lift force. One can see that there are fluctuations of aerodynamic forces.

In order to analyze the influences of aerodynamic forces on the relaxation factor, the average standard deviation is introduced for evaluating the fluctuation of aerodynamic forces. The average standard deviation is described as

$$
S=\sqrt{\frac{1}{n-5} \sum_{i=3}^{n-2}\left(f_{i}-\overline{f_{i}}\right)^{2}}
$$

where

$$
\bar{f}_{j}=\frac{1}{5} \sum_{j=j-2}^{j+2} f_{j} \quad j=3,4, \ldots, n-2,
$$

where $n$ is the total number of time steps, and $\bar{f}_{i}$ is the aerodynamic force at time $i$ obtained by the five-point mean method.

Fig. 5 shows the average standard deviation of drag force, side force, lift force, roll moment, pitch moment and yaw moment with different relaxation factors. When the relaxation factor equals 0.5 , the average standard

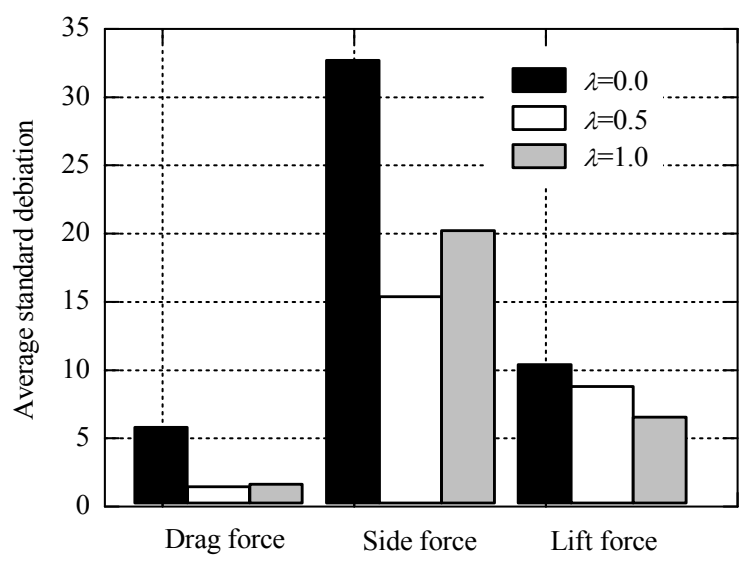

(a) Forces

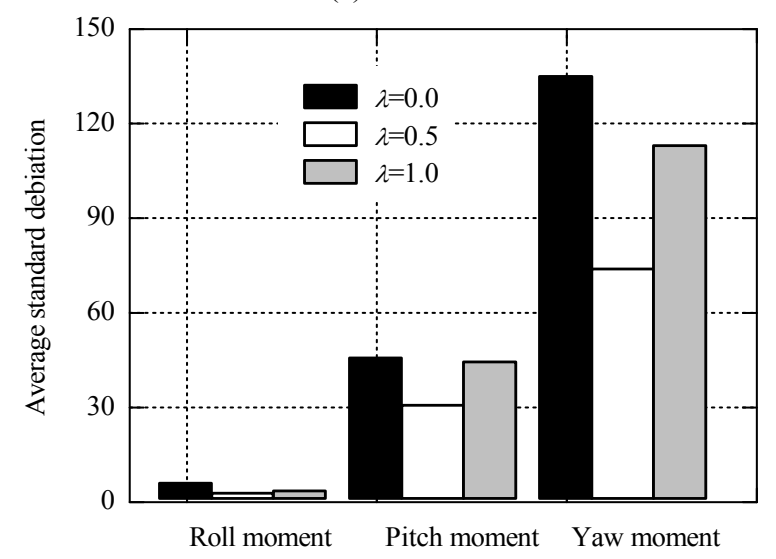

(b) Moments

Fig. 5 Standard deviation of aerodynamic forces 
deviation of force or moment is nearly the minimum in the three cases. That means the fluctuations of aerodynamic forces are relatively smaller.

In addition, another definition of standard deviation of error is introduced for evaluating the error between the predicted aerodynamic forces and the calculated aerodynamic forces. The standard deviation of error is described as

$$
\bar{S}=\sqrt{\frac{1}{n-2} \sum_{i=2}^{n-1}\left(f_{i}-\tilde{f}_{i}\right)^{2}} .
$$

Fig. 6 shows the standard deviation of error of drag force, side force, lift force, roll moment, pitch moment and yaw moment with different relaxation factors. Similarly, when relaxation factor equals 0.5 , the standard deviation of error of force or moment is nearly the minimum in the three cases. From Eq. (13), one knows that the predicted aerodynamic forces are more close to the calculated aerodynamic forces.

By comparing average standard deviation and standard deviation of error of the aerodynamic forces calculated with different relaxation factors, one can see that the fluctuation of aerodynamic forces is small and that

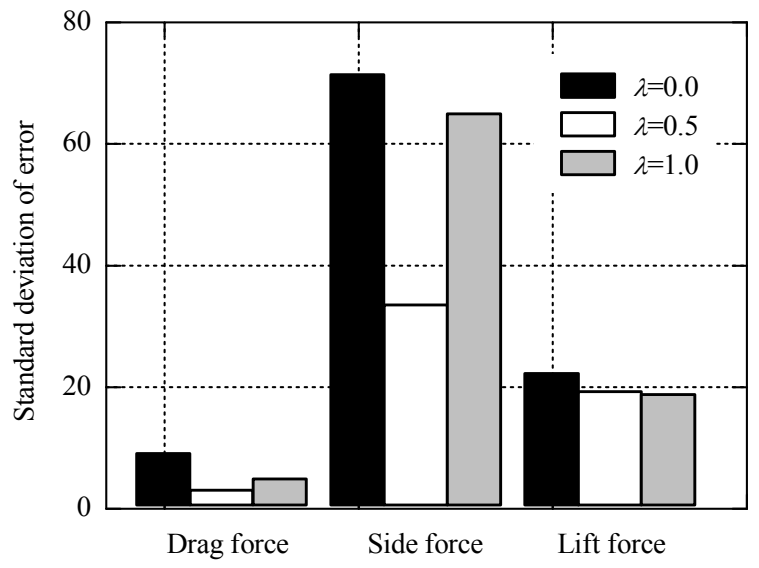

(a) Forces

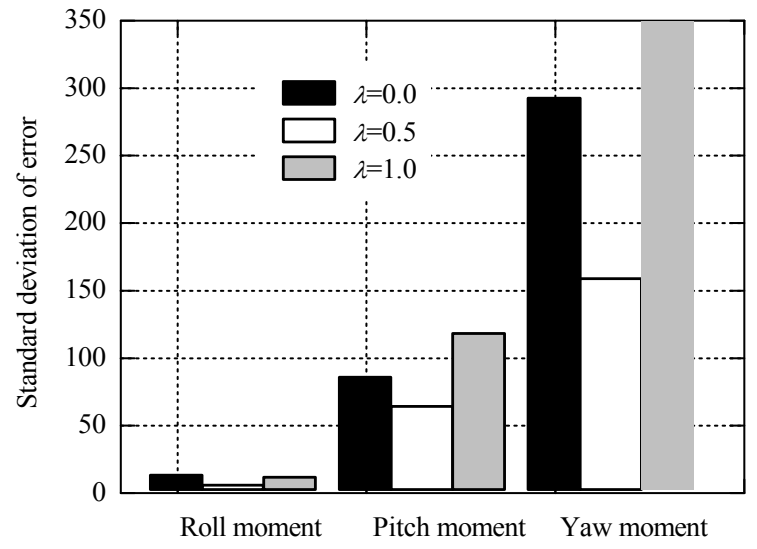

(b) Moments

Fig. 6 Standard deviation of error of aerodynamic forces the predicted aerodynamic forces are closer to the calculated aerodynamic forces when the relaxation factor equals 0.5 .

\subsection{Fluid-structure interaction dynamics}

Fig. 7 shows the pressure distribution of a high-speed train cross-section. The cross-section is $14 \mathrm{~m}$ away from the nose of the head train. We can see that, under the interaction effect, the pressure of the train windward side becomes larger and the pressure on the lee side becomes smaller. In addition, the pressure of the train bottom becomes larger because it has turned into lee side after the roll of the train body.

The side force comparison of the head train is shown in Fig. 8. The side force calculated with off-line simulation is a steady value; however, the one with cosimulation shows a fluctuant curve. After considering the interaction effect, the side force becomes larger than before. The side force is closely related to the pressure difference between the lee side and the windward side. According to the analysis of pressure distribution around the train cross-section, as shown in Fig. 8, the pressure difference becomes larger after taking the change of

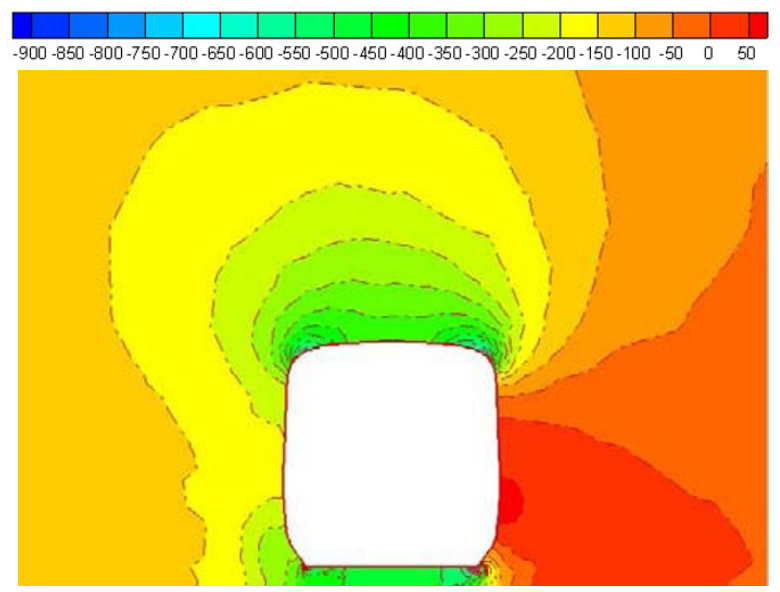

(a) Off-line simulation

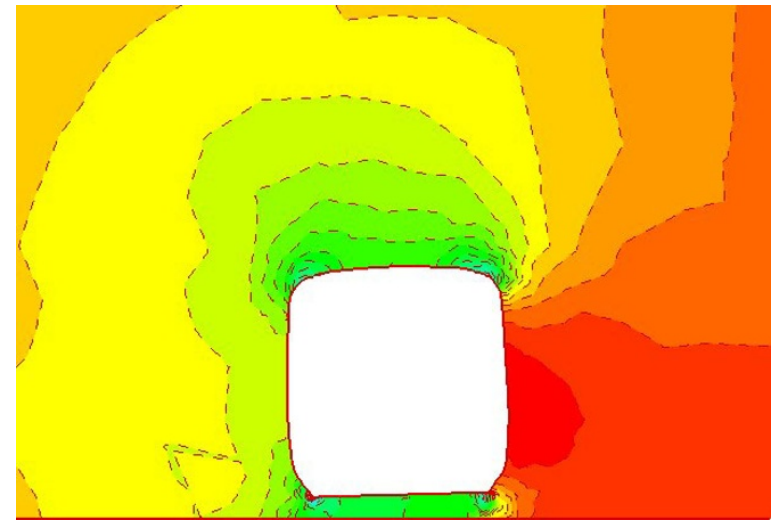

(b) Co-simulation

Fig. 7 The pressure distribution of cross-section 
train attitude into account. Thus, the side force calculated with co-simulation is over $10 \%$ more than that with off-line simulation.

The train-track coupling dynamic responses to the aerodynamic forces are calculated. There are some differences in the dynamic responses because of the differences of forces and moments. Fig. 9 shows the lateral displacement comparison of the head train. After we take the interaction effect into account, the lateral displacement of the head train toward the lee side is larger than before. However, the variations of lateral displacement are similar as the lateral displacement mainly depends on the lateral track irregularity.

Table 1 shows the comparison of train security indexes calculated by different methods. After considering the interaction effect, the security indexes, including the wheel/rail vertical force, lateral wheelset force, de-

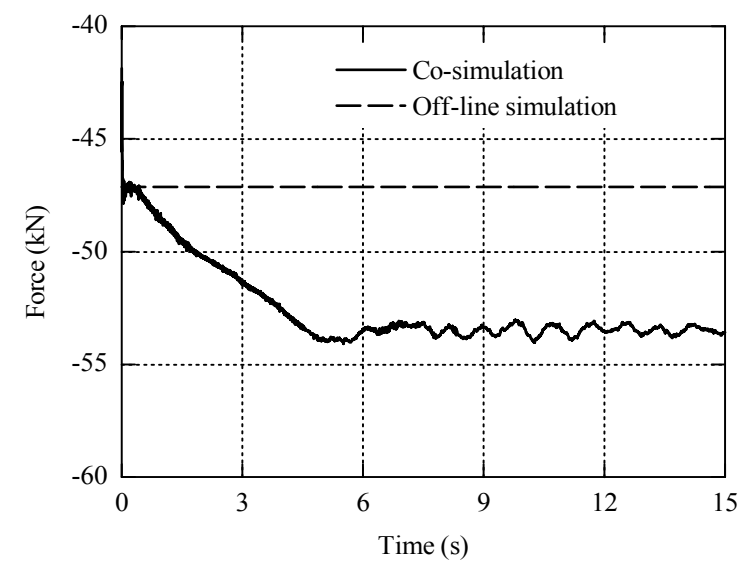

Fig. 8 Side force comparison of head train

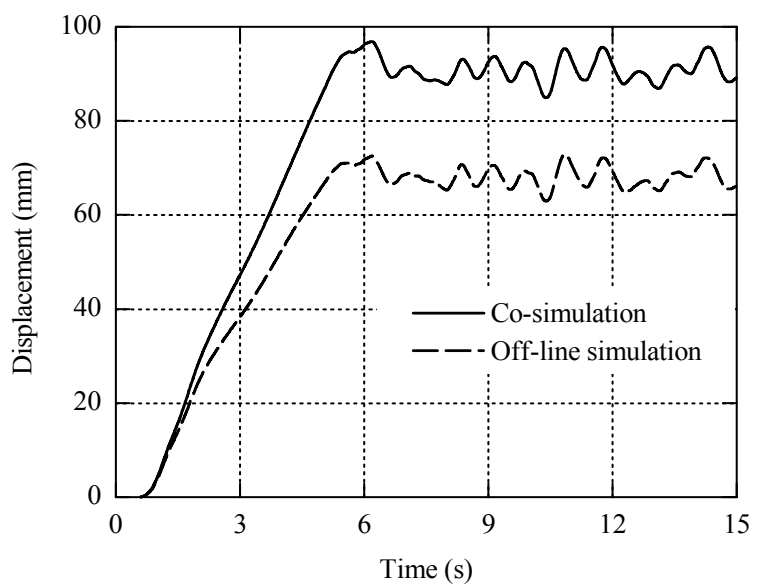

Fig. 9 Lateral displacement comparison of head train

Table 1 Comparison of train security indexes

\begin{tabular}{ccccc}
\hline Method & $\begin{array}{c}\text { Wheel/rail } \\
\text { vertical } \\
\text { force }(\mathrm{kN})\end{array}$ & $\begin{array}{c}\text { Lateral } \\
\text { wheelset } \\
\text { force }(\mathrm{kN})\end{array}$ & $\begin{array}{c}\text { Derail- } \\
\text { ment }\end{array}$ & $\begin{array}{c}\text { Wheel } \\
\text { unloading }\end{array}$ \\
\hline Off-line & 94.69 & 28.79 & 0.31 & 0.63 \\
Co-simulation & 98.92 & 32.85 & 0.35 & 0.78 \\
\hline
\end{tabular}

railment, and wheel unloading, become larger. This means that the fluid-structure interaction cannot be neglected in the calculation of train security.

\section{Conclusion}

In this paper, an improved algorithm of high-speed train fluid-structure interaction under crosswind is presented. First, data communication of the fluid solver and structure solver is avoided by inserting the program of the train-track coupling dynamics into the fluid dynamics program; second, the load boundary of the fluidstructure interface is improved by introducing the relaxation factor. For the velocity of crosswind of $13.8 \mathrm{~m} / \mathrm{s}$ and a running speed of train at $350 \mathrm{~km} / \mathrm{h}$, the aerodynamic forces and attitude of the head train are compared via the off-line simulation and the cosimulation. The comparison shows that the fluidstructure interaction has a significant influence on the head aerodynamics and attitude, and the security indexes become larger in the fluid-structure interaction simulation. Thus, the fluid-structure interaction calculation is necessary for high-speed trains under crosswind conditions.

\section{Acknowledgements}

The research was supported by the National Natural Science Foundations of China (Nos.50821063 and 50823004), 973 Program (No.2007CB714701) and the Fundamental Research Funds for the Central Universities (No.2010XS34).

\section{References}

[1] X.B. Li, Z. Yang, J.Y. Zhang, W.H. Zhang, Aerodynamics properties of high-speed train in strong wind, Journal of Traffic and Transportation Engineering, 2009, 9(2): 66-73 (in Chinese).

[2] H.Q. Tian, Study development of train aerodynamics in China, Journal of Traffic and Transportation Engineering, 2006, 6(1): 1-9 (in Chinese).

[3] M. Suzuki, K. Tanemoto, T. Maeda, Aerodynamic characteristics of train/vehicles under cross winds, Journal of Wind Engineering and Industrial Aerodynamics, 2003, 91: 209-218.

[4] C.J. Baker, J. Jones, F. Lopez-calleja, et al, Measurements of the cross wind forces on trains, Journal of Wind Engineering and Industrial Aerodynamics, 2004, 92(7): 547-563.

[5] S.G. Tan, X.B. Li, Z. Yang, J.Y. Zhang, et al, The flow field structure and the aerodynamic performance of high speed trains running on embankment under cross wind, Rolling Stock, 2008, 46(8): 4-8 (in Chinese). 
[6] B. Dierichs, M. Sima, A. Orellano, et al. Crosswind stability of a high-speed train on a high embankment, Journal of Rail of Rapid Transit, 2007, 221(2): 205-225.

[7] Y.G. Wang, K. Chen, Effects of crosswinds on curve negotiation of high-speed power cars, Journal of Southwest Jiaotong University, 2005, 40(2): 224-227 (in Chinese).

[8] Y. Song, Z.S. Ren, Research on dynamics performance of high speed trains under strong lateral wind, Rolling Stock, 2006, 44(10): 4-7 (in Chinese).

[9] T. Li, J.Y. Zhang, W.H. Zhang, Performance of vehicletrack coupling dynamics under crosswinds, In: Proceeding of Railway Motor Vehicle Dynamic Simulation,
Chengdu, 2010: 317-322 (in Chinese).

[10] J.Z. Yang, H.Q. Bi, W.M. Zhai, Dynamic analysis of train in cross-winds with the arbitrary LagrangianEulerian Method, Journal of the China Railway Society, 2009, 31(2): 120-124 (in Chinese).

[11] T. Cui, W.H. Zhang, Study on safety of train in side wind with changing attitudes, Journal of the China Railway Society, 2010, 32(5): 25-29 (in Chinese).

[12] W.M. Zhai, Two simple fast integration methods for large-scale dynamic problems in engineering, International Journal for Numerical Methods in Engineering, 1996, 39(24): 4199-4214.

(Editor: Dongju CHEN) 